\title{
Enjeu du collectif - noms collectifs en jeux
}

Résumé : En partant du postulat que certains jeux de mots se basent sur des propriétés remarquables de la langue, cet article s'intéresse à des cas de jeux de mots dans lesquels le collectif et la pluralité ont une place centrale. Les énoncés étudiés proviennent de la littérature, de la publicité et principalement de la presse. Ils comportent des noms collectifs, des expressions au singulier ou au pluriel défini ; c'est à partir de ce matériau linguistique que le jeu de mots se construit, en exploitant ses caractéristiques morphosyntaxiques (singulier morphologique), sémantiques (double niveau du nom collectif) et interprétatives (appréhension collective et distributive). Avec ces exemples, on discute les limites de la notion de jeu de mots, en défendant l'idée que le jeu de mots correspond à un plaisir de l'intelligence, mais n'est pas nécessairement drôle.

Mots clés : ambigüité, collectif, distributif, générique, nom collectif, pluralité, pluriel, singulier, synecdoque

\section{Introduction}

Cet article porte sur les jeux de mots, ou les jeux avec les mots dont le procédé essentiel repose sur la pluralité, le collectif, ou encore sur le rapport entre singulier et pluriel, d'un point de vue grammatical et sémantique. Premier échantillon illustratif, les exemples (1) à (5) présentent tous cette mise en «balance », cette articulation entre le collectif, c'est-à-dire une appréhension globale (p. ex. groupe, peuple, multitude) et l'individu, ou entre singulier et pluriel, exprimée de différentes manières : mise en relation frontale par la structure syntaxique, le lexique, les reprises anaphoriques entre collectif et pluralité d'individus en (1), (2) et (3) et entre global et disséminé en (4) et (5).

(1) [critique de La petite foule, livre de Christine Angot] Dans ce portrait de groupe avec individus ${ }^{1}$, on devine parfois l'auteure. Attentive et sensible. (Télérama, 05/03/2014)

1 Sans doute une allusion à la version française du titre du roman de Heinrich Böll Gruppenbild mit Dame, paru en 1971. 
(2) [critique de La petite foule, livre de Christine Angot] Fondue parmi les mille visages de cette multitude qu'elle dépeint, Christine Angot n'est ici omniprésente que d'une façon [...]. (Télérama, 05/03/2014)

(3) [guerre du Kosovo] Nous avons imaginé ce que fut le calvaire des Kosovars [...] en regardant les visages innombrables de ce peuple en larmes. (Libération, 11/06/1999, éditorial)

(4) [dans une salle de tribunal] Et l'innombrable regard de la salle confirmait. (Daniel Pennac, Monsieur Malaussène, Paris : Gallimard, 1997)

(5) [À propos du temps de travail] Jamais l'Amérique n'a connu une aussi longue période de prospérité, et jamais, à en croire les statistiques, elle n'a autant passé de temps au travail. (Le Nouvel Observateur, 02-08/11/2000, titre : «Le ras-le-bol des Américains »)

Je pars ici du postulat que les jeux de mots prennent place dans les potentialités de jeu (au sens large) que la langue ou le discours offrent, et que, par voie de conséquence, certains jeux de mots peuvent être des révélateurs de questions linguistiques et également, à ce titre, être rapprochés d'autres effets de sens qu'on ne peut pas réellement qualifier de « jeux de mots ». Le lien entre jeux de mots et certains phénomènes linguistiques spécifiques est souvent souligné, notamment à propos de l'homonymie, de l'homophonie (voir p.ex. Rittaud-Hutinet 2014), de la paronymie (p.ex. Jeandillou 2009 et à paraitre), ou encore de la polysémie, avec des jeux de mots comme le calembour, la syllepse, l'antanaclase (voir p.ex. Guiraud 1976 ; Rémi-Giraud 2006 ; Rullier-Theuret 2015 ; Knospe 2015 ; Rabatel 2015). ${ }^{2}$

Pour ma part, je touche plutôt ici à des questions concernant la grammaire et le lexique, que je regroupe provisoirement (en me limitant au français) sous le terme de «collectif». Les données que je présente ici proviennent d'une recherche plus générale sur les noms collectifs (Ncoll), d'où il ressort que ces types de noms ont des propriétés régulières remarquables, d'un point de vue morphosyntaxique, et surtout sémantique - et notamment une discordance entre un singulier morphologique et une pluralité sémantique, et des potentialités d'appréhension globale (collective) ou des individus (distributive). ${ }^{3}$

Certaines des propriétés grammaticales ou sémantiques des Ncoll apparaissent aussi avec d'autres catégories, avec des effets de sens (ludiques, rhétoriques) comparables, ce qui m'incite à rassembler des formes certes différentes

2 Pour un aperçu général, voir aussi les contributions du Discussion forum dans Knospe, Onysko et Goth (éds.) (2016), et en particulier Winter-Froemel, Thaler et Lecolle.

3 Pour ces termes et leur emploi à propos des Ncoll et des syntagmes définis pluriel, voir notamment Martin (1984), Kemmer (1993), Lecolle (2001 et inédit) et Mihatsch (2015). 
(noms propres de lieu, syntagmes définis pluriel et Ncoll) mais ayant des effets proches. Comme d'autres catégories du vocabulaire (toponymes, ou encore adjectifs relationnels), ils ont ce que j'appelle une «épaisseur sémantique » : ils sont, par exemple, propices aux glissements de sens et aux ambigüités ${ }^{4}$.

L'article présentera donc la rencontre entre des faits linguistiques et des jeux de mots ou des jeux avec les mots dont la plupart, pour être ludiques, ne sont pas pour autant drôles : ils se situent donc dans une zone dont la validité doit être débattue. Dans une première partie $(\S 2)$, je présenterai la conception du jeu de mots sur laquelle je m'appuie, en défendant l'idée qu'un jeu de mots peut ne pas être drôle. Dans une deuxième partie $(\S 3)$, je définirai et caractériserai les types de phénomènes linguistiques qui sont à la base des jeux de mots relevés. Je décrirai ensuite ces exemples (§4), avant de faire un retour, en conclusion, sur les limites des données analysées en termes de jeux de mots.

\section{Caractérisation des jeux de mots : parti-pris et choix}

Si les jeux de mots sont, du moins dans la tradition française, généralement associés à l'humour et à la drôlerie ${ }^{5}$, on peut en réalité tout à fait les en dissocier. Les jeux de mots ont en commun avec l'humour d'être une manifestation d'intelligence et d'une certaine virtuosité, comme le signalent notamment Arnaud, Maniez, et Renner (2015), à partir des travaux d'Attardo. Mais l'humour ne se limite pas aux manifestations de langage, et se situe dans une perspective plus large.

De leur côté, les jeux de mots opèrent centralement à partir de matériau verbal. Comme le dit (en français) Rullier-Theuret (2015 : 39), se basant sur Hausmann $^{6}$, « Le jeu de mots en tant que manipulation rhétorique doit, comme toutes des manipulations, se signaler, c'est-à-dire il doit s'arranger pour que l'auditeur le remarque en tant que manipulation ». On peut en citer plusieurs caractéristiques :

\footnotetext{
4 Pour ce qui concerne la notion d'ambigüité, les différences entre ambigüité et malentendu et les types d'ambigüité, je me réfère aux travaux de Fuchs (voir Fuchs 1986, 1991, 1996).

5 Voir Guiraud (1976).

6 "Das Wortspiel ist nur dann Wortspiel, wenn es sich in irgendeiner Weise signalisiert ». Et : « Das Wortspiel aber setzt vor seine Manipulation die Warnung : Vorsicht, ich manipuliere! » (Hausmann $1974: 16$ ).
} 
- le jeu de mots nécessite plusieurs mots et donc des relations entre les mots relations in praesentia ou in absentia ;

- il consiste en une manipulation du matériau verbal du point de vue de la forme et du point de vue du sens. La forme, donc, est cruciale ;

- il opère dans le cadre d'un empan resserré. Certains auteurs parlent de "forme raccourcie», de "force de compression », et les jeux de mots généralement donnés en exemple ont cette caractéristique ;

- le jeu de mots est conscient et volontaire, et il se donne à voir comme tel.

Ceci n'implique donc pas que le jeu de mots soit nécessairement amusant, même s'il l'est souvent. D'ailleurs, ni jeu ni ludique dans leur définition ne contiennent nécessairement l'idée d' « amusant », mais plutôt celle de gratuité, de surplus.

Tout en ayant en commun avec l'humour la manifestation de surplus et d'intelligence, les jeux de mots peuvent donc être non drôles, comme ceux qu'on trouve dans les titres de livres ou de films - (6) -, dans certaines publicités - (7) et (8) -, dans des sentences et des aphorismes - (9) -, qui, au mieux, font sourire mais, surtout, produisent une jouissance intellectuelle ou esthétique :

(6) Peuples en larmes, peuples en armes (Didi-Huberman, 2016)

(7) Funtastisch (slogan publicitaire pour la montre Swatch, cf. Knospe 2015)

(8) Un mal, des mots (Slogan de SOS-Amitié, cf. Lecolle 2016)

(9) Nous ne naissons pas seuls. Naître, pour tout, c'est connaître. Toute naissance est une connaissance. (Paul Claudel, Traité de la co-naissance au monde et de soi-même, Euvre poétique, cf. Winter-Froemel 2016 ; Lecolle 2016)

Jacqueline Henry qui, cherchant à définir le jeu de mots, discute cette question, mentionne elle aussi la virtuosité, la jouissance intellectuelle ou spirituelle :

il semble que la fonction ludique des jeux de mots tienne non pas à ce qu'ils font rire, mais à ce qu'ils distraient ou divertissent, au sens premier de ces termes ; autrement dit, ils détournent l'esprit d'un sens donné pour en faire surgir un autre [...]. (Henry 2003 : 35).

Le point de vue que j'adopte ici est en continuité avec ces propositions, et accepte dans la catégorie générale « jeux de mots » des séquences drôles ou non, du moment qu'elles ont les caractéristiques citées. Ce point de vue ne s'intéresse pas centralement à l'intention initiale de production d'un jeu de mots ni à sa réus-

7 Voir Winter-Froemel (2016). 
site. ${ }^{8}$ Toutefois, dans la mesure où, comme dit supra, le jeu de mots « se signale ", on peut considérer que, de facto, les jeux de mots dont on dispose sont ceux qui ont réussi, du moins à réception, et ce quelle que soit l'intention initiale.

J'adopte donc une conception des jeux de mots qui ne se limite pas à ce qui est drôle ou destiné à l'être. Ce choix est également lié au type de données recueillies : les jeux de mots analysés sont tirés de titres de livres ou d'articles de presse, dont on sait qu'il s'agit d'un genre accueillant pour les jeux de mots. ${ }^{9}$ Ils proviennent également d'extraits de textes de presse, par exemple dans des éditoriaux et parfois en chute d'article, où le genre textuel prescrit de terminer par ce qu'on appelle « un bon mot ». Enfin, certains proviennent de la publicité ou de slogans politiques.

Non conçus pour être « drôles », ils n'en sont pas moins « remarquables », du point de vue formel et sémantique. Il ne s'agit pas ici de manipulation touchant la face sonore des mots associés - comme pour les allitérations ou les paronomases - ni de défigement, mais le travail sur la forme se situe plutôt dans le cadre de relations syntagmatiques : dans un empan réduit, ces jeux de mots mettent en place des oppositions, des antithèses, des paradoxes, ou encore des relations de composition inattendues ou remarquables, comme en (3) ou en (4). Le jeu se développe donc, non pas à partir de sonorités ou de propriétés graphiques, mais à partir de phénomènes touchant centralement la reconnaissance $\mathrm{du} / \mathrm{des}$ sens des segments, et leur mise en présence - ce pour quoi on peut hésiter à les qualifier de jeux de mots, de jeux sur les mots, de jeux avec les mots. ${ }^{10}$

L'analyse des séquences prend place dans le cadre plus large de la description des effets de sens des Ncoll : sans chercher en définitive une distinction tranchée entre jeux de mots et jeux avec les mots, on cherche donc à situer le jeu dans le cadre plus général de faits discursifs dans lesquels les propriétés linguistiques des Ncoll ont un rôle central.

8 L'intention est néanmoins à mettre en lien avec le type de discours et le genre textuel où apparait le jeu de mots, et parfois avec la place où apparait la réalisation ludique dans le texte (paratexte, chute d'article pour ce qui concerne mes données).

9 Pour les titres de presse, voir les travaux de Sullet-Nylander (notamment Sullet-Nylander 2005, et la contribution de l'auteure dans ce volume).

10 Les caractéristiques exposées ici ne cadrent pas avec ce qui permet de distinguer jeu de mots et divertissement linguistique (jeu avec les mots) chez Guiraud (1976 : 98-101). 


\section{Noms collectifs et collectif}

Pour comprendre les spécificités des effets de sens mentionnés, cette section introduit les caractéristiques des Ncoll et du collectif.

\subsection{Définition et description des noms collectifs (en français)}

Un Ncoll est un nom au singulier qui désigne une entité constituée d'un ensemble d'individus de même catégorie. ${ }^{11}$ En voici quelques exemples :

Humanité, foule, peuple, jeunesse, public, fratrie, personnel, groupe, gouvernement, opinion publique.

Plusieurs faits caractérisent les Ncoll (je me centre sur ce qui est pertinent par rapport à la réalisation de jeux de mots) :

- du point de vue morphologique, ils sont au singulier, ce qui introduit une discordance avec leur sens qui, lui, est pluriel ;

- du point de vue sémantique, ils sont structurés en deux niveaux, deux « unités » : celle du tout, global, et celle de ses éléments, les individus, envisagés dans leur pluralité.

L'existence de ce double niveau a de nombreuses incidences, et notamment le fait que le groupe lui-même d'un côté, les éléments qui le composent de l'autre ont une certaine indépendance l'un par rapport à l'autre : créer ou changer un groupe, lui donner un nom en tant que groupe, le qualifier sont des processus qui ne portent pas du tout ou pas directement sur les éléments.

\subsection{Noms collectifs et effets de sens}

En tant que dénomination, au singulier, d'un groupe constitué - c'est-à-dire d'un tout - et d'une pluralité d'éléments, le Ncoll se prête spécifiquement à des effets de sens, qui peuvent reposer sur :

- la constitution du groupe ;

- la partition du groupe (rapport partie/tout) ;

11 Voir (principalement) Lammert (2010) et Lammert et Lecolle (2014), ainsi que Lecolle (inédit) pour les Ncoll humains. 
- les relations entre les parties du groupe (comparaisons ou oppositions, somme des parties) ;

- la relation entre le groupe et les éléments qui le composent (les individus);

- la composition plus ou moins précise ou précisable du groupe, c'est-à-dire l'extension du nom, du point de vue quantitatif ou qualitatif (combien d'éléments ? quels éléments ou quels types d'éléments ?);

- la pluralité ou la grande pluralité en tant que telle (multitude, horde, foule),

caractéristiques sémantiques auxquelles s'ajoute, sur les plans morphosyntaxique et sémantique, la distorsion, en français, entre pluralité sémantique et singulier morphologique.

Toutes ces caractéristiques ne donnent pas lieu à des jeux de mots. Mais elles constituent un terreau dans lequel ceux-ci peuvent s'ancrer. Dans les exemples présentés précédemment et par la suite, c'est, notamment, la dualité que constituent le niveau du groupe dans sa globalité et le niveau des individus dans leur pluralité qui est en cause - en jeu.

\subsection{Noms collectifs et « collectif » comme catégorie}

Le collectif est représenté, sur le plan du lexique, principalement par les Ncoll. ${ }^{12}$ Au-delà, d'autres types de réalisations nominales présentent des potentialités sémantiques similaires, sur un plan ou un autre. Récapitulons : le collectif, au sens où je l'entends c'est-à-dire la dualité entre une appréhension globale, cohésive et une pluralité, peut se réaliser en français :

- dans les Ncoll (une pluralité abordée par un singulier grammatical) ;

- dans d'autres noms à sens collectif : des noms de lieux comme salle ou des toponymes comme Amérique en emploi métonymique - voir (4) et (5);

- dans les expressions plurielles (je me limite aux groupes nominaux définis au pluriel - les brebis, par exemple) ;

- dans les pronoms anaphoriques (en, ou des pronoms personnels), qui interviennent comme relais - elle en (5).

12 Le collectif n'est pas délimité de la même façon selon les langues ; il peut également être une catégorie flexionnelle (voir Mihatsch 2015). Au sein du lexique, il touche différentes parties du discours, et notamment le nom et le verbe (voir Kemmer 1993). Je limite mon analyse au nom et au groupe nominal. 
Par ailleurs, ces problématiques concernant le singulier/pluriel (et donc la globalité/dissémination) et leurs rapports rencontrent aussi, pour le français, la question du sens générique, lorsqu'il est exprimé par un groupe nominal défini pluriel ou singulier - les hommes ou l'homme, pour dire « un homme quel qu'il soit », paraphrase elle-même proche sémantiquement de «tous les hommes ». Certains de mes exemples de jeux de mots comportent donc l'expression du sens générique. ${ }^{13}$

\subsection{Référence totus / Référence omnis}

L'opposition mentionnée en titre de cette section porte sur une des manifestations de la dualité entre le niveau du tout et le niveau des éléments, celle qui oppose l'appréhension du groupe en tant que tel, de manière globale (référence totus) à l'appréhension des éléments de manière distributive (référence omnis). Cette dualité, définitoire des Ncoll, se manifeste également avec les groupes nominaux définis pluriel. Commençons par un exemple sous forme de blague, une devinette, qui me permet d'illustrer cette distinction - à effet potentiellement ludique. Elle est tirée d'un article de Robert Martin qui traite des déterminants définis pluriel en emploi générique (1984).

[...] «- Quelles sont les brebis qui mangent le plus ? Les blanches ou les noires ?

- Les blanches parce qu'elles sont en plus grand nombre.»

La subtilité de cette "devinette» vient justement de ce que la réponse, collective, y a quelque chose d'étonnant. La question posée orientait fortement vers une réponse distributive (par exemple « Les brebis blanches mangent plus que les brebis noires parce qu'elles sont plus corpulentes »). (Martin 1984 : 190)

La réponse porte sur un aspect quantitatif de l'appréhension collective, ici le total. C'est le total des brebis noires, d'un côté et blanches de l'autre qui sont comparés, alors que la question "quelles sont ? " laissait attendre une réponse sur les caractéristiques individuelles des brebis noires et des brebis blanches comparativement.

En se rapprochant davantage des jeux de mots, (10) fournit une autre illustration de la dualité omnis / totus, avec une dénomination au pluriel. Plus qu'un

13 Parmi les nombreux travaux en sémantique du français portant sur la question de la référence générique et de son expression, voir les travaux de Michel Galmiche (notamment Galmiche 1985), de Georges Kleiber (notamment Kleiber 1988), et de Robert Martin - voir infra Martin (1984). 
réel jeu de mots, l'énoncé est un clin d'œil, qui met en évidence un paradoxe linguistique.

(10) Le 100ème anniversaire des Petits chanteurs à la croix de bois [...] Visages d'anges et voix de cristal, ils parcourent le monde en chantant et bossent dur pour y arriver. Les Petits chanteurs à la croix de bois ont 100 ans. Émission hommage, avec de "vraies " stars pour les accompagner. (Télérama, 20/12/2006, programme télévision)

Bien que grammaticalement au pluriel, l'expression polylexicale Les Petits chanteurs à la croix de bois fonctionne en bloc, puisqu'il s'agit du nom du groupe de chanteurs. De son côté, le groupe verbal ont cent ans, bien qu'accordé au pluriel, se rapporte au groupe de chanteurs lui-même en tant que groupe constitué, et non pas aux individus - éléments du groupe - lesquels n'ont naturellement pas « cent ans », mais sont (éternellement) des enfants, qui se succèdent au sein du groupe.

La plupart des exemples de jeux de mots collectés ici reposent d'une manière ou d'une autre sur cette dualité entre tout constitué et ensemble d'individus. Ils permettent de mettre en évidence une sorte de hiatus entre forme et sens, puisqu'une même forme (une expression plurielle ou collective) peut avoir plusieurs sens, sans qu'on puisse pour autant parler d'ambigüité, au sens où deux interprétations de même niveau d'analyse (lexique, syntagme, phrase) seraient mutuellement exclusives (voir Fuchs 1996) : on a affaire à une polyvalence, et c'est par là que s'engouffre le jeu, mais parfois aussi la mécompréhension, le malentendu, ou encore les réalisations fallacieuses ou ayant un rôle argumentatif.

\section{Jeux de mots et jeux avec les mots basés sur le " collectif » : des exemples}

Dans les jeux de mots présentés à l'analyse, il faut souligner la part fondamentale que prennent des faits sémantiques liés intrinsèquement au collectif : les rapports de constitution entre partie et tout ou entre élément et collection sont tout particulièrement impliqués via le lexique, la pluralité morphologique comme en (10) ou au contraire le singulier, et mis en évidence par la forme, c'est-à-dire ici par la mise en relation syntagmatique des séquences concernées.

À vrai dire, il est assez difficile de classer les exemples, car ils mettent tous en jeu plusieurs questions touchant à ces relations de sens. Dans ce qui suit, je propose néanmoins une structuration, en commençant par le plus simple. Dans 
les cas où je dispose d'exemples non ludiques, ils seront présentés en parallèle, afin de mettre en évidence le phénomène linguistique à l'œuvre.

\subsection{Rapports de constitution du groupe et « pluralité de pluralité ॥}

Voici un premier cas : il s'agit de jeux reposant sur des expressions de rassemblement, de dispersion, ainsi que des expressions qui mentionnent le changement. Dans les exemples concernés, la structure en deux niveaux de sens du Ncoll est à la source d'une ambigüité potentielle : lorsqu'il est question d'un changement, parle-t-on du changement du groupe ? Ou au sein du groupe?

Ces faits apparaissent, en particulier, en combinaison avec la pluralité - une pluralité de pluralités en réalité, puisqu'à la pluralité sémantique lexicale du Ncoll s'ajoute le pluriel morphologique - auquel correspond également une pluralité sémantique. C’est pour ces raisons que (11) est potentiellement ambigu, même s'il est non ludique :

(11) Votre musique, qui emprunte autant à la chanson qu'à l'électro ou au rap, réconcilie les générations. (Télérama, interview du chanteur belge Stromae, 04/09/2013)

S’agit-il de réconcilier entre elles les différentes générations ? Ou les éléments de chaque génération ? Ou une combinaison de tout cela?

Voyons maintenant (12) que, cette fois, je considère comme ludique. L'énoncé vient en titre d'un article présentant un lieu associatif de Metz, «la Maison d'Anjou », qui « accompagne parents et enfants pour favoriser les échanges et le développement de l'enfant au sein du foyer » (présentation).

(12) 20 années de réunions de familles (en titre d'un article du journal municipal de Metz, mai 2014).

Le jeu de mots repose sur un jeu de pluralité et un détournement phraséologique : d'abord parce qu'habituellement, dans cette expression, famille est au singulier (organiser / participer à une réunion de famille) ; ensuite parce que réunion prend dans cet énoncé un sens processuel : réunions de familles est à interpréter comme « on réunit des familles ». Le jeu repose enfin sur le Ncoll famille : s’il est mis au pluriel, il devient potentiellement ambigu pour les mêmes raisons qu'en (11). En réalité, le jeu de mots exhibe plutôt une dualité d'interprétations possibles, sans qu'il y ait une réelle ambigüité : qu'est-ce qui est réuni, chaque famille ou les familles entre elles? 
En (13), le ressort ludique est de nouveau la pluralité interne de la France, et la répartition de cette pluralité.

(13) La France est divisée en quarante millions de Français (cité par Guiraud 1976 : 11).

Divisée est, certes, polysémique, mais sa signification est « poussée » hors de ses limites ordinaires : en principe, elle se rapporte aux parties d'un tout, mais non aux éléments ultimes que sont, ici, les citoyens : le jeu substitue à une constitution du tout en parties (sens de diviser) une constitution de ce tout en éléments.

\section{2 Éléments et collectif en relation de composition}

Dans les exemples qui suivent, éléments et collection, l'un composant l'autre, sont mis en relation syntagmatique.

Cette relation est présente en (14)-(15), par le biais de la copule, dans une sorte de choc frontal entre le pluriel disséminé (les paysans, ils) et le global du collectif (espèce, l’Algérie) :

(14) Les paysans sont une espèce en voie de disparition. (Télérama, 03-09/02/2001).

(15) Ils ont moins de 40 ans, ils sont l'Algérie d'aujourd'hui (radio années 90, en introduction du sujet).

Dans une deuxième série - (2)-(4) et (16) ci-dessous -, le global et la pluralité des éléments sont mis en relation de manière abrupte par la structure en $\left[\mathrm{N}_{1}\right.$ de $\left.\mathrm{N}_{2}\right]$, où $\mathrm{N}_{2}$ exprime cette globalité par un Ncoll (ou par un nom à référence métonymique collective - salle) et $\mathrm{N}_{1}$ la pluralité, parfois quantitativement amplifiée par un adjectif (mille visages, innombrable regard, visages innombrables). On a un effet d'hyperbole, qui repose tout autant sur cette amplification que sur l'effet « disséminateur » de la référence distributive $\left(\mathrm{N}_{1}\right.$ au pluriel, lequel dénote une fonction humaine individuelle - regard, voix - ou une partie du corps - visage).

(2) [critique de La petite foule, livre de Christine Angot] Fondue parmi les mille visages de cette multitude qu'elle dépeint, Christine Angot n'est ici omniprésente que d'une façon [...]. (Télérama, 05/03/2014)

(4) [dans une salle de tribunal] Et l'innombrable regard de la salle confirmait. (Daniel Pennac, Monsieur Malaussène, Paris : Gallimard, 1997)

(3) [guerre du Kosovo] Nous avons imaginé ce que fut le calvaire des Kosovars [...] en regardant les visages innombrables de ce peuple en larmes. (Libération, 11/06/1999, éditorial) 
(16) Les voix du peuple : l'espace public sonore au XVIII (titre d'un entretien de l'historienne Arlette Farge, 23/05/2012, à propos de l'ouvrage Essai pour une histoire des voix au dixhuitième siècle, Bayard, 2009)

En (16), intervient en outre la polysémie de peuple : en effet, alors que la première interprétation conduit vers "le peuple» comme entité politique pris dans sa globalité, il est question dans l'entretien des voix individuelles des membres du peuple, mais dans l'autre acception de ce nom : le «petit » peuple, c'est-à-dire les classes populaires (voir Tamba 2012).

\section{3 Éléments et collectif en opposition}

Dans cette section sont présentés des cas où les éléments et le collectif sont mis en « balance », et parfois en opposition. En voici tout d'abord deux exemples non ludiques: (17) et (18) énoncent une construction intellectuelle dans le cadre d'une argumentation développée et structurée, dans laquelle un élément central est, précisément, la mise en opposition entre le collectif, global, et la pluralité des éléments.

(17) Aux XVIII ' ou XIX ${ }^{e}$ siècles, l'humanité était encore une abstraction ; aujourd'hui, c'est devenu une réalité : tous les hommes sont sur le même bateau. Ils sont condamnés à l'intérêt général! Mais il n'est pas facile de le construire. [...] (Télérama, interview de Catherine Larrère, philosophe, 15/07/2015)

(18) La jeunesse ? parlons plutôt des jeunes, dans toute leur diversité sociale et culturelle, répond l'historienne Ludivine Bantigny, prompte à balayer quelques préjugés [...]. (Télérama interview 15/10/2014)

Les exemples qui suivent sont en revanche, selon moi, des jeux de mots ou des jeux sur les mots : on y trouve une sorte de «clin d'œil », de jeu stylistique et existentiel raffiné et un peu gratuit.

(1) [critique de La petite foule, livre de Christine Angot] Dans ce portrait de groupe avec individus, on devine parfois l'auteure. Attentive et sensible. (Télérama, 05/03/2014)

(19) Faute d'éradiquer la pauvreté, on déracine les pauvres (Médiapart, juillet 2013, en titre, à propos d'expulsions de Roms)

(20) Nicolas Sarkozy fait disparaître l'opposition, non les mécontents. (radio, début 2008)

Est-on dans le jeu de mots ? Dans le jeu sur ou avec les mots? 
Quoi qu'il en soit, ces jeux ont une finalité autre : celle de mettre en évidence une contradiction, un paradoxe, en se basant sur le rapport de composition et en en jouant. En (20) par exemple, les mécontents sont supposés (approximativement) composer l'opposition. En (19), on perçoit le lien morphologique et sémantique entre pauvre et pauvreté : d'un point de vue sémantique, la pauvreté est une caractéristique constitutive du pauvre - la personne pauvre. Si l'on va plus loin et qu'on donne, par synecdoque, à pauvreté le sens de "l'ensemble des pauvres $»^{14}$ - ce qui n'est pas son sens, mais qui pourrait l'être comme dans le cas de jeunesse («qualité d'être jeune » et « ensemble des jeunes ») -, l'effet de sens est encore un renforcement de cette antithèse existentielle.

\subsection{Jeux singulier/pluriel, générique, et appréhension totus / omnis}

Les exemples qui suivent ont en commun de mettre en jeu l'articulation du singulier et du pluriel. En général, le singulier grammatical renvoie au global et le pluriel à la dispersion, la dissémination ; mais la pluralité n'est elle-même pas toujours exprimée par un pluriel grammatical, et inversement le pluriel grammatical ne s'oppose pas à une appréhension singulière et cohésive (voir (10)). Il en résulte que plusieurs phénomènes se combinent et que plusieurs niveaux d'interprétation sont intriqués.

Même si un autre axe aurait été possible, ma présentation rassemble les exemples selon ce point de vue sémantique. Par le biais de réalisations «ludiques » ou du moins remarquables du point de vue de leur forme, cette présentation permet de mettre en évidence, ici encore, le hiatus entre une forme (p. ex. l'expression du singulier ou du pluriel) et un sens (individuel, pluriel, global, générique).

Les énoncés ou fragments textuels exposés peuvent être décrits comme des jeux de « zoom », allant de la focalisation sur des éléments dans leur pluralité à une vision globale, un " grand angle ". Certains cas se situent au-delà, dans le domaine du trope, dans la mesure où l'usage des mots et des structures plurielles est " poussé » en dehors des limites normales, voire renversé. Le mécanisme à l'œuvre est alors généralement à classer dans le champ de la synecdoque : audelà la diversité de synecdoques relevées par la tradition ${ }^{15}$, on postule un même

14 Voir Meyer (1993).

15 Voir Dumarsais (1730); Meyer (1993, 1995). 
principe, propice à ces jeux de zoom, celui de mettre en jeu la partie et le tout, le plus et le moins (sur le plan de l'extension et de l'intension).

(5) [À propos du temps de travail] Jamais l'Amérique n'a connu une aussi longue période de prospérité, et jamais, à en croire les statistiques, elle n'a autant passé de temps au travail. (Le Nouvel Observateur, 02-08/11/2000, titre : « Le ras-le-bol des Américains »)

L'Amérique dénote tout d'abord le pays (les États-Unis) du point de vue économique et politique. Dans la suite, le pronom elle en reprise a une valeur de pluriel à cause du prédicat dont il est sujet - c'est bien chaque individu (chaque Américain actif) qui passe du temps au travail, et en même temps c'est la somme de ces individus qui peut être intégrée dans des décomptes et des statistiques. Parallèlement, en tant que forme au singulier, le pronom elle conserve iconiquement une portée globale.

Outre le jeu avec le singulier et le pluriel et avec le collectif, on touche ainsi, dans cet exemple et dans les suivants, le domaine du générique avec les expressions au singulier - un combattant en (21), l'œil en (23), le citoyen en (24), lui en (25) - , c'est-à-dire, sur le plan sémantico-discursif, le domaine dans lequel l'individu désigné « vaut pour » tous ceux qui pourraient être impliqués ou représentés. Ces jeux entrent en composition également avec celui portant sur le global et le disséminé qu'on a déjà vu précédemment. Outre la morphologie (singulier / pluriel) et la syntaxe, le sens lexical y intervient - cf. innombrable en (21).

(21) [...] tous les coups sont, non seulement permis, mais exigés par ce type de guerre qui consiste à éradiquer (le mot a d'ailleurs fait fortune) un combattant innombrable et camouflé [...]. (Jean Lacouture, Télé'Obs, juillet 1998. À propos d'un film sur la guerre du Vietnam)

(21) représente un procédé en quelque sorte inverse à celui de (22), exemple classique de synecdoque (le pluriel sémantique pour le singulier) :

(22) Il a reçu la visite du corps médical [à propos d'un seul médecin]. (Cité par Meyer 1993 : 158)

En (23), le jeu syntagmatique avec le singulier (l'œil) et le pluriel (des indigènes) dans un même groupe nominal met en scène la représentativité d'une partie du corps d'un individu (l'œil, employé métonymiquement pour « le regard ») « valant pour » tous les indigènes - voir en comparaison (23') où la métonymie demeure, mais où est perdue la valeur de représentativité de l'individu pour le groupe.

(23) [à propos d'un livre qui se passe en Malaisie] le romancier s'est servi de l'œil des indigènes pour regarder les Blancs. (critique de Malaisie de Henri Fauconnier. Le Figaro Littéraire, 17/12/1998) 
(23') Mais le romancier s'est servi des yeux des indigènes pour regarder les Blancs.

En (24), dans la partie entre crochets, le jeu entre singulier morphologique à sens générique (au citoyen, le citoyen) et pluralité, par le biais du groupe verbal (a déjà versé 34 milliards), vient en chute d'une argumentation portant sur la grève au sein d'une entreprise publique (Air France) et ses conséquences, notamment en termes d'impôt pour les citoyens. Tout l'extrait est basé sur cette balance entre singulier générique et singulier renvoyant à une pluralité, entre global et dissémination.

(24) La grève dans le secteur public ne pénalise pas l'employeur, mais toute la population. Infliger, grâce à la grève, une sanction financière au patron quand celui-ci est l'État, c'est pénaliser les citoyens. Ce qui fausse le jeu, c'est que, dans tous les conflits du travail où le patron est l'État, les deux parties se mettent d'accord sur le dos du tiers absent qui est aussi le tiers payant, c'est-à-dire le citoyen. La grève ne coûte rien à l'État patron. Elle ne coûte rien aux salariés de l'État. [Elle ne coûte qu'au citoyen. Le citoyen a déjà versé 34 milliards pour permettre à Air France de continuer]. (Jacques Julliard, Nouvel Observateur, 11/06/1998, à propos d'une grève des pilotes d'Air France)

L'exemple (25) correspond au texte d'une affiche du Comité Chrétien contre la Faim et pour le Développement (CCFD), qui alerte sur le fléau de la vente d'armes dans des pays en guerre où la population est pauvre. De par son objectif (sensibiliser) et son support (affiche), le texte est proche de la formule publicitaire. S’il se rapproche, sur le plan thématique, du slogan politique, il est néanmoins plus long et complexe - et illustre même assez nettement la virtuosité dont il était question en section 2.

(25) En 2000 on lui a versé 34 milliards de francs pour l'aider à vivre et on lui a vendu 45 milliards de francs d'armes pour l'aider à mourir (affiche CCFD, début des années 2000)

(25) reproduit le seul texte de l'affiche, complété par une photo représentant un homme jeune tenant une arme et bardé de munitions. C'est cet homme qui est désigné par les pronoms lui et l' dans le texte. Mais cet homme est naturellement là comme représentant singulier d'une population - représentant comme indivi$\mathrm{du}$ « mis pour » une pluralité, mais aussi comme individu générique - le membre " par excellence » d'une population -, égale et identique aux autres (comme en (23) avec l'œil, la métonymie en moins). Les syntagmes verbaux (versé 34 milliards de francs et vendu 45 milliards de francs d'armes) ne peuvent vraisemblablement, comme en (24), que renvoyer à un collectif (un État, une organisation) et non un individu (lui). On a donc là, avec le matériau verbal et la photo, un rapport complexe entre singulier et pluralité, et mettant en œuvre toutes les dimensions sémantiques de cette singularité et de cette pluralité. 


\section{Conclusion}

Les données que j'ai exposées et discutées dans le cadre de cette étude sur les jeux de mots proviennent d'une recherche plus générale sur les noms collectifs (plus précisément les noms collectifs humains, voir Lecolle inédit). Comme on l'a $\mathrm{vu}$, ces noms ont des propriétés remarquables sur le plan morphosyntaxique, et surtout sémantique (lien entre un tout et les éléments qui le composent, combinaison de singulier et de pluriel). L' « épaisseur sémantique » qui en résulte les rend particulièrement susceptibles d'entrer dans des configurations discursives, spécifiques du point de vue textuel mais aussi rhétorique et communicationnel.

Il me faut maintenant faire un retour sur les exemples présentés, en posant la question de leur statut : comment les traiter, dans le cadre des jeux de mots? Autrement dit : s'agit-il ou non de jeu de mots, ou de jeu avec/sur les mots?

Mes analyses mettent le sémantique au centre : les jeux de mots y reposent étroitement sur la face "signifié » des signes linguistiques concernés - si l'on veut bien considérer la valeur sémantique des catégories singulier, pluriel, collectif, générique -, et de leurs relations. De son côté, le travail de la forme intervient par le biais des relations syntagmatiques et, plus précisément, syntaxiques : structures prédicatives avec un sujet au singulier et un prédicat à sens pluriel ; groupe nominal en $\left[\mathrm{N}_{1}\right.$ de $\mathrm{N}_{2}$ ] ou [N Adj] confrontant singulier et pluriel, global et disséminé.

Par ailleurs, on reconnait dans les exemples présentés une attention portée aux mots et aux relations entre mots, un réel travail d'écriture et donc une intention manifeste. Mais ces jeux de mots ne font pas rire - ce n'est d'ailleurs pas leur but. Ils peuvent être au service d'une fonction d'appel ou de présentation, comme dans les titres de presse ou les introductions de sujet radio ou dans les slogans publicitaires. Souvent aussi, ils entrent dans une argumentation, par exemple comme chute d'un raisonnement, dans les éditoriaux journalistiques ou les tribunes, où ils contribuent à renforcer cette argumentation.

En définitive, si l'on doit répondre à la question : " jeu de mots ou non ? », on posera d'abord qu'il y a des cas où l'on ne peut pas trancher. Ensuite, voici quelques arguments : outre la question de l'intention et du travail des mots - qui ne sont, en réalité, pas spécifiques au jeu de mots -, plusieurs facteurs me paraissent déterminants, et surtout la combinaison de ces facteurs : dans le cadre englobant d'un lien sémantique entre mots, la mise en évidence d'une combinaison surprenante (entre singulier et pluriel, entre global et disséminé) ou d'une opposition (la jeunesse/les jeunes), et ceci d'une manière directe. Ces faits ne peuvent intervenir que dans un format resserré, et c'est bien ce trait, dont on a vu qu'il constitue un critère pour les jeux de mots, qui me parait décisif. 
En résumé, donc, voici ce qui caractérise les jeux de mots / jeux avec les mots présentés : épaisseur sémantique, hiatus ou contradiction intrinsèque entre forme et sens, et exhibition de ces traits par un dispositif syntaxique mettant en relation frontale les mots concernés.

\section{Références bibliographiques}

Arnaud, Pierre J. L., François Maniez \& Vincent Renner. 2015. Non-Canonical Proverbial Occurrences and Wordplay : A Corpus Investigation and an Enquiry Into Readers' Perception of Humour and Cleverness. In Angelika Zirker \& Esme Winter-Froemel (éds.), Wordplay and Metalinguistic / Metadiscursive Reflection. Authors, Contexts, Techniques, and MetaReflection (The Dynamics of Wordplay 1), 135-159. Berlin \& Boston : De Gruyter.

Didi-Huberman, Georges. 2016. Peuples en larmes, peuples en armes. Paris : les Éditions de Minuit.

Dumarsais, César Chesneau. [1730] 1988. Des tropes ou des différents sens. Présentation, notes et traduction Françoise Douay-Soublin. Paris : Flammarion, collection «Critiques ».

Fuchs, Catherine. 1986. Le vague et l'ambigu : deux frères ennemis. Quaderni di semantica 7(2). 235-245.

Fuchs, Catherine. 1991. L'hétérogénéité interprétative. In Herman Parret (éd.), Le sens et ses hétérogénéités, 107-120. Paris : Éditions du CNRS.

Fuchs, Catherine. 1996. Les ambiguïtés du français. Gap-Paris : Ophrys.

Galmiche Michel. 1985. Phrases, syntagmes et articles génériques, Langages 79. 2-39.

Guiraud, Pierre. 1976. Les Jeux de mots. Paris : Presses Universitaires de France, coll. «Que sais-je ?».

Hausmann, Franz Josef. 1974. Studien zu einer Linguistik des Wortspiels. Das Wortspiel im "Canard enchaîné ». Tübingen : Niemeyer.

Henry, Jacqueline. 2003. La traduction des jeux des mots. Paris : Presses de la Sorbonne Nouvelle.

Jeandillou, Jean-François. 2009. «Accepter qu'un texte puisse se porter tout seul » : le prétexte onomastique dans les Vœux de Perec. Poétique 157. 41-52.

Jeandillou, Jean-François. À paraitre. Gangue maternelle et tangage châtié. Une littérature de jeunesse au risque ludique de la dyslexie. In Bettina Full \& Michelle Lecolle (éds.), Jeux de mots et créativité : langue(s), discours et littérature (The Dynamics of Wordplay 4). Berlin \& Boston : De Gruyter.

Kemmer, Suzanne. 1993. Marking oppositions in Verbal and Nominal Collectives. Faits de langues 2. 85-95.

Kleiber, Georges. 1988. Phrases génériques et raisonnement par défaut. Le Français moderne 56(1-2). 1-15.

Knospe, Sebastian. 2015. A cognitive model for bilingual puns. In Angelika Zirker \& Esme Winter-Froemel (éds.), Wordplay and Metalinguistic / Metadiscursive Reflection. Authors, Contexts, Techniques, and Meta-Reflection (The Dynamics of Wordplay 1), 161-193. Berlin \& Boston : De Gruyter. 
Knospe, Sebastian, Alexander Onysko \& Maik Goth (éds.). 2016. Crossing Languages to Play with Words : Multidisciplinary Perspectives (The Dynamics of Wordplay 3). Berlin \& Boston : De Gruyter.

Lammert, Marie. 2010. Sémantique et cognition : les noms collectifs. Genève-Paris : Droz.

Lammert, Marie \& Michelle Lecolle. 2014. Les noms collectifs en français, une vue d'ensemble. Cahiers de Lexicologie 105(2). 203-222.

Lecolle, Michelle. 2001. Figures et référence plurielle, en corpus journalistique. Cahiers de Grammaire 25. 29-52.

Lecolle, Michelle. 2016. Some specific insights into wordplay form : sublexical vs lexical level. In Sebastian Knospe, Alexander Onysko \& Maik Goth (éds.), Crossing Languages to Play with Words : Multidisciplinary Perspectives (The Dynamics of Wordplay 3), 63-70. Berlin \& Boston : De Gruyter.

Lecolle, Michelle. Inédit. Noms collectifs humains en français : enjeux sémantiques, lexicaux et discursifs.

Martin, Robert. 1984. Les usages génériques de l'article et la pluralité. In Jean David \& Georges Kleiber (éds.), Déterminants : syntaxe et sémantique, Actes du Colloque International de linguistique organisé par la Faculté de Lettres et de Sciences Humaines de Metz, 187-202. Paris : Klincksieck.

Meyer, Bernard. 1993. Synecdoques, étude d'une figure de rhétorique Vol. 1. Paris : Éditions l'Harmattan.

Meyer, Bernard. 1995. Synecdoques, étude d'une figure de rhétorique Vol. 2. Paris : Éditions l'Harmattan.

Mihatsch, Wiltrud. 2015. Collectives. In Peter O. Müller, Ingeborg Ohnheiser, Susan Olsen \& Franz Rainer (éds.), HSK Word-Formation. An International Handbook of the Languages of Europe, 1183-1195. Berlin : De Gruyter Mouton.

Rabatel, Alain. 2015. La plurisémie dans les syllepses et les antanaclases. Vox Romanica 74. 124-156.

Rémi-Giraud, Sylviane. 2006. Du calembour à la création sémantique : en passant par la syllepse.... In Yannick Chevalier \& Philippe Wahl (éds.), La Syllepse, figure stylistique, 121138. Lyon: Presses Universitaires de Lyon.

Rittaud-Hutinet, Chantal. 2014. Équivoque homophonique en français : polyvalence fortuite et ambiguïté volontaire. Études romanes de Brno 35(1). 141-161.

Rullier-Theuret, Françoise. 2015. Calembours bons et jeux de mots laids chez San-Antonio. In Brigitte Buffard-Moret (éd.), Bons mots, jeux de mots, jeux sur les mots : de la création à la réception, 27-40. Arras : Artois Presses Université.

Sullet-Nylander, Françoise. 2005. Jeux de mots et défigements à la Une de Libération (19732004). Langage et société 112.111-139.

Tamba, Irène. 2012. « Le peuple » : un nom collectif, une notion ambivalente. In Michel Wieviorka (éd.), Le peuple existe-t-il ?, Les entretiens d'Auxerre, 17-26. Auxerre : Éditions Sciences Humaines.

Thaler, Verena. 2016. Varieties of Wordplay. In Sebastian Knospe, Alexander Onysko \& Maik Goth (éds.), Crossing Languages to Play with Words : Multidisciplinary Perspectives (The Dynamics of Wordplay 3), 47-62. Berlin \& Boston : De Gruyter.

Winter-Froemel, Esme. 2016. Approaching Wordplay. In Sebastian Knospe, Alexander Onysko \& Maik Goth (éds.), Crossing Languages to Play with Words : Multidisciplinary Perspectives (The Dynamics of Wordplay 3). 11-46. Berlin \& Boston : De Gruyter. 